\title{
Detection of Pyuria by Microscopic Urinalysis as a Marker of Pediatric Urinary Tract Infection
}

Dhiraj Shrestha ${ }^{1}$, Pratigya Thapa ${ }^{2}$, Dinesh Bhandari ${ }^{3}$, Balkrishna Bhattachan ${ }^{4}$, Hiramani Parajuli ${ }^{5}$, Prakash Chaudary ${ }^{5}$, Vijay Kumar Sharma ${ }^{6}$, Pradeep Kumar Shah ${ }^{5}$

${ }^{1}$ Department of Microbiology, Shi-Gan International College of Science and Technology, Kathmandu, Nepal.

2Department of Microbiology, Kathmandu UpatyakaKhanepani Limited (KUKL), Kathmandu, Nepal. ${ }^{3}$ School of Public Health, University of Adelaide, Australia.

${ }^{4}$ Biotechnology Society Nepal, Bhaktapur, Nepal.

${ }^{5}$ Department of Microbiology, Tri-Chandra Multiple College, Kathmandu, Nepal.

${ }^{6}$ Department of Biochemistry, Institute of Medicine, TUTH, Kathmandu, Nepal.

\begin{abstract}
Globally, different diagnostic tests of urinary tract infection (UTI) are in clinical practices. A reliable test can increase the efficiency of the healthcare system, especially in a developing country like Nepal, reducing cost and time. Thus, we accessed the possibility of pyuria detected by microscopic urinalysis as a marker of pediatric UTI. The prospective study was conducted fromJuly2014 to January 2015 at Alka hospital, Lalitpur. Microscopic urinalysis of 353clean-catch urine samples was done by the wet mount method, followed by urine culture by a semiquantitative method. We confirmed $64(18.1 \%)$ UTI cases by culture, the gold standard for UTI diagnosis. Fever was the most common clinical manifestation in UTI cases. The sensitivity, specificity, positive predictive value and negative predictive value of pyuria detected by microscopic urinalysis to identify UTI were $50 \%, 70.9 \%, 27.6 \%$ and $86.5 \%$ respectively. In 318 febrile cases, the sensitivity, specificity, positive predictive value and negative predictive value of pyuria detected by microscopic urinalysis to identify UTI were $73.2 \%, 72.6 \%, 28.3 \%$ and $94.8 \%$ respectively. The findings suggest pyuria detected by microscopic urinalysis as not a worthwhile marker of pediatric UTI. But it is a trust worthy marker in febrile pediatric cases.
\end{abstract}

Keywords: febrile, marker, microscopic urinalysis,pediatrics, pyuria, UTI

*Corresponding Author

Email: balkrishnabhattachan@gmail.com

\section{Introduction}

Urinary tract infection (UTI) is a common infection in all age groups [1-3] and affects at least $1 \%$ of boys and $3 \%$ of girls[4].UTI is difficult to diagnose in children, as symptoms are non-specific [5-7].Complications of UTI in children lead to renal scarring and terminal kidney damage[8].UTI management varies with evolving research findings[8].The diagnosis of UTI should base clinically and confirmed by urine culture [9]. Urine culture is a gold standard for the diagnosis of UTI, but it takes up to $24 \mathrm{hrs}$ for final reporting [10]. Using microscopic urinalysis allows starting antimicrobial treatment 24 hours sooner than waiting for culture results [11]. Microscopic urinalysis can thus be a useful test for the rapid diagnosis of UTI in children [10]. But, no single cut-off count of leucocytes exhibits high sensitivity and specificity $[5,12]$. At least 5 leucocytes per high power field (HPF) of centrifuged urine is commonly considered as pyuria [13]. Pyuria is mostly observed as a result of inflammation, thus it is a common sign of UTI [14]. This makes pyuria a suitable marker of UTI. This study aimed to access the utility of microscopic urinalysis as a potential marker to diagnose pediatric UTI.

\section{Materials and Methods}

The cross-sectional prospective descriptive study was conducted from July 2014 to January 2015 at Alka Hospital, Lalitpur, Nepal. The ISO 9001:2008 accredited hospital is a referral hospital at Kathmandu valley. A total of 8,692 urine samples were submitted to the microbiology laboratory for culture during the study period. Only 353 non-repetitive, clean catch urine samples from infants and children patients, under 13 years of age and with symptoms of UTI, were included in the study. 
Table 1. Clinical symptoms in patients

\begin{tabular}{lcc}
\hline Symptoms & $\begin{array}{c}\text { Suspected } \\
\text { UTI cases } \\
\text { (\% of 353 } \\
\text { cases) }\end{array}$ & $\begin{array}{c}\text { Confirmed } \\
\text { UTI cases } \\
\text { (\% of } 64 \\
\text { cases) }\end{array}$ \\
\hline $\begin{array}{l}\text { Abdomen } \\
\text { pain }\end{array}$ & $212(60.1)$ & $33(51.6)$ \\
$\begin{array}{l}\text { Dysuria } \\
\text { Fever }\end{array}$ & $233(66.0)$ & $32(50.0)$ \\
$\begin{array}{l}\text { Frequency } \\
\text { of urine }\end{array}$ & $222(62.9)$ & $32(50.0)$ \\
$\begin{array}{l}\text { Malodorous } \\
\text { urine }\end{array}$ & $71(20.1)$ & $46(71.9)$ \\
\hline
\end{tabular}

The symptoms were abdomen pain and/or dysuria and/or fever and/or frequency of urine and/or malodorous urine. For infants and younger children, symptoms were fever and parental reporting of malodorous urine. The children who were already on antibiotics therapy were excluded. The clean-catch urine samples were collected in a sterile container. In infants and non-toilet-trained children, a sterile plastic bag was attached to genitalia for clean catch urine collection. In toilet-trained children, voided midstream urine sample was collected. Each sample was first subjected to microscopic urinalysis by the wet mount method. In brief, $10 \mathrm{~mL}$ of urine was centrifuged at $3000 \mathrm{rpm}$ for $5 \mathrm{~min}$. The supernatant was discarded, and the sediment was re-suspended in $500 \mu L u$ urine.

This native urine sediment was dropped on a glass slide and covered by a coverslip. The microscopic examination was performed by the bright-field microscopy $(x 400)$. The threshold value of at least 5 pus cells/HPF, which corresponds to at least 25 leukocytes per $\mathrm{mL}$ of non-centrifuged urine, was considered as pyuria[13]. In parallel, each sample was subjected to urine culture by a semi-quantitative method. In brief, $1 \mu \mathrm{L}$ urine was streaked on MacConkey agar (HiMedia Ltd, India) and blood agar plate (HiMedia Ltd, India) using a calibrated loop of $2 \mathrm{~mm}$ size. Growths were observed after 18-48hrs of aerobic incubation at $37^{\circ} \mathrm{C}$. The growth of at least 100 colonies on the agar plate, which corresponds to at least $10^{5}$ colony-forming units (CFU) per $\mathrm{mL}$ of urine, were considered as culture-positive [15]. Data were entered and stored using Microsoft Excel (version 2010, Microsoft Corporation, USA). Chi-square test of variables was performed whenever applicable and $p$ values below 0.05 were considered significant.

\section{Results}

The mean age of patients was $5 \pm 3.5$ years (ranging from 1 month to 12 years; variance= 12.5). In our study, the male to female ratio of UTI suspected cases was 1:1.4. UTI was confirmed by culture in $64(18.1 \%)$ out of 353 patients. Meanwhile male to female ratio of UTI confirmed cases was 1:1.2.Fever was the most common clinical symptom in UTI confirmed cases, 49 (76.6\%) followed by malodorous urine, 46 (72\%) (Table 1).

In $18(62.1 \%)$ of 29 males and $14(40 \%)$ of 35 females who were confirmed of UTI did not have pyuria (Table 2).

Of 64 UTI cases, 32 (50\%) casesshowed pyuria and $32(50 \%)$ cases did not show pyuria. This was statistically significant since pyuria was associated with an increased risk of bacteriuria $(\mathrm{p}<0.05)$ (Table 3).

Table 2: Number of pus cells/HPF and bacteriuria in male and female patients

\begin{tabular}{cccccc}
\hline \multirow{2}{*}{ Pus cells/HPF } & \multirow{2}{*}{$\begin{array}{c}\text { No. of } \\
\text { sample }\end{array}$} & $\begin{array}{c}\text { Culture } \\
\text { negative }\end{array}$ & $\begin{array}{c}\text { Culture } \\
\text { positive }\end{array}$ & $\begin{array}{c}\text { Culture } \\
\text { negative }\end{array}$ & $\begin{array}{c}\text { Culture } \\
\text { positive }\end{array}$ \\
\hline$<3$ & 181 & 70 & 14 & 86 & 11 \\
$3-5$ & 56 & 23 & 4 & 26 & 3 \\
$5-8$ & 39 & 13 & 3 & 21 & 2 \\
$8-10$ & 14 & 2 & 2 & 9 & 1 \\
$10-15$ & 20 & 5 & 1 & 12 & 2 \\
$\geq 15$ & 43 & 7 & 5 & 15 & 16 \\
\hline Total & 353 & 120 & 29 & 169 & 35 \\
\hline
\end{tabular}


Table 3: Relationship between microscopic urinalysis and culture in all suspected cases

\begin{tabular}{|c|c|c|c|}
\hline \multirow{2}{*}{ Pyuria } & \multicolumn{2}{|c|}{ Urine culture } & \multirow{2}{*}{ Total $(\%)$} \\
\hline & Culture positive (\%) & Culture negative $(\%)$ & \\
\hline Pyuria & $32(50)$ & $84(29.1)$ & $116(32.9)$ \\
\hline Nonpyuria & $32(50)$ & $205(70.9)$ & $237(67.1)$ \\
\hline Total & $64(100)$ & $289(100)$ & $353(100)$ \\
\hline $\begin{array}{l}\text { Sensitivity }=50 \% \\
\text { Specificity }=70.9 \% \\
\text { Positive predictive } \\
\text { Negative predictiv }\end{array}$ & $\begin{array}{l}\mathrm{u}=27.6 \% \\
\text { lue }=86.5 \%\end{array}$ & & \\
\hline \multicolumn{4}{|c|}{ Table 4: Relationship between microscopic urinalysis and culture in febrile cases } \\
\hline $\begin{array}{l}\text { Pyuria in febrile } \\
\text { cases }\end{array}$ & \multicolumn{2}{|c|}{ Urine culture } & Total (\%) \\
\hline Pyuria & $30(73.2)$ & $76(27.4)$ & $106(33.3)$ \\
\hline Nonpyuria & $11(26.8)$ & $201(72.6)$ & 212(66.7) \\
\hline Total & $41(100)$ & $277(100)$ & $318(100)$ \\
\hline $\begin{array}{l}\text { Sensitivity }=73.2 \% \\
\text { Specificity }=72.6 \% \\
\text { Positive predictive } \\
\text { Negative predictiv }\end{array}$ & $\begin{array}{l}\text { ue }=28.3 \% \\
\text { lue }=94.8 \%\end{array}$ & & \\
\hline
\end{tabular}

Of 41 febrile UTI cases, only $30(73.2 \%)$ cases showed pyuriaand $11(26.8 \%)$ did not show pyuria. This was statistically significant since pyuria was associated with an increased risk of bacteriuria in febrile cases $(\mathrm{p}<0.05)$ (Table 4$)$

\section{Discussion}

UTI can only be accurately diagnosed by a combination of clinical and laboratory investigations. Wide ranges of practices are seen among physicians [16]. Over diagnosis of UTI had been a common problem that had led to aggressive antibiotic therapy [1].

We first classified the pool of 353 cases, as UTI and non-UTI, depending on the culture. Thus, $18.1 \%$ of cases were confirmed to have UTI. The discordance of clinical and laboratory investigations could have resulted in low growth positivity. In our study, male to female ratio of suspected cases was 1:1.4, this was involuntary recruitment bias. Meanwhile male to female ratio of UTI confirmed cases was 1:1.2. The natural epidemiology pattern of UTI shows more prevalence in females[17].The urethra of females are colonized with colonic Gramnegative bacteria as they are shorter in length and are in close proximity to the anus, thus females are more frequently affected by UTI [18]. In our study, fever was the common symptom, manifested in $76.6 \%$ of UTI cases.
This was similar to reports from other studies [19-21].

There is no unison in the cut-off value of pus cells to consider as pyuria. The cut-off value of $\geq 5$ pus cells/HPF was considered pyuria[13]. Out of total 237 samples without pyuria, $13.50 \%$ were culture positive; and of 116 samples with pyuria, $27.6 \%$ were culture positive. The relationship of pyuria and culture was statistically significant $(\mathrm{p}<0.05)$. Culture positive without pyuria often occurs in patients with diabetes, enteric fever of bacterial endocarditis whereas pyuria with sterile culture occurs in patients with prior antibiotic use, renal tuberculosis, corticosteroid administration, analgesic nephropathy, or renal calculi [18]. In our study, since no distinction of samples from patients was made on these criteria, both bacteriuria without pyuria and pyuria without bacteriuria may have occurred. The sensitivity, specificity, positive predictive value and negative predictive value of pyuria to diagnose UTI were $50 \%, 70.9 \%, 27.6 \%$ and $86.5 \%$ respectively. This was slightly lower but comparable with reports from other studies [19, 22-24]. Our study revealed pyuria with less sensitivity and high specificity. This finding indicates that the presence of pyuria may not suggest UTI but the absence of pyuria can exclude UTI. Furthermore, positive predictive 
value and negative predictive value suggest that using pyuria to diagnose UTI in children will result in a significantly larger number of falsepositive and lower false-negative results. Therefore our study suggests that pyuria detected by microscopic urinalysis is a less reliable marker for pediatrics UTI but can be used to exclude UTI as a single test modality. Some authors still agree that microscopic urinalysis can identify only a third to half of the patients with positive urine culture [25-27].

We further accessed the reliability of pyuria detected using microscopic urinalysis by dividing the study population based on the presence or absence of symptom fever to improve predictive scores. Out of total 318 febrile cases, 9.4\% were culture positive along with pyuria and $3.5 \%$ samples were culture positive without pyuria. The relationship of pyuria and culture in febrile cases was statistically significant $(\mathrm{p}<0.05)$. Thus, in febrile cases, the sensitivity, specificity, positive predictive value and negative predictive value of pyuria to diagnose UTI increased to $73.2 \%$, $72.6 \%, 28.3 \%$ and $94.8 \%$ respectively. Our study revealed pyuria with higher sensitivity and specificity in febrile cases. This finding indicates that the presence of pyuria in febrile cases can suggest UTI; similarly, the absence of pyuria in febrile cases can exclude UTI. Furthermore, low positive predictive value and high negative predictive value suggest that using pyuria to diagnose UTI in febrile children can result in a higher number of false-positive but lower falsenegative results. This suggests that pyuria detected by microscopic urinalysis is a worthwhile marker for UTI in febrile children.

Furthermore, our study suggests pyuria detected by microscopic urinalysis can serve as a reliable marker of UTI in pediatrics in a primary healthcare setting where prevalence is much lower. This can omit unnecessary tests, thus can increase effective diagnosis and cost in the healthcare system. Nitrate reduction test and leucocyte esterase (LE) test as recommended by the National Institute for Health and Care Excellence (NICE) can further be used to improve this diagnosis accuracy [5].
Nevertheless, our study doesn't underrate the importance of culture for UTI diagnosis in children. But reliable marker can increase the effectiveness of diagnosis excluding unnecessary tests. Critical cases need a quick diagnosis for prompt treatment that cannot wait culture result which usually demands 18-48 hours. In an economy lagged country like Nepal, this can help to improve and outreach the healthcare facility especially in a primary healthcare system where there is a fundamental lack of enough resources for investigations; and treatment is primarily based on clinical suspicion. Thus, pyuria detected by microscopic urinalysis can be a standalone diagnostic test in febrile cases in such settings.

\section{Conclusion}

Our findings suggest pyuria detected by microscopic urinalysis entertain less sensitivity and specificity, thus pyuriais not the reliable marker of UTI in pediatrics. However, the reliability of the pyuria detected by microscopic urinalysis was higher to diagnose UTI in febrile pediatric cases, which can be a single test model in low resource settings like the primary healthcare system.

\section{Conflict of Interest}

None declared

\section{Acknowledgments}

None

\section{Consent to publish}

Not applicable

\section{Ethical approval and consent to the participant}

The study was a laboratory-based study and a part of the study was a routine patient care investigation. No patient-related data were collected except the demographic parameters, thus ethical approval was not required. Oral informed consent was taken from a guardian on behalf of the patients.

\section{Availability of data and materials} All data generated or analyzed during this study are included in the article. Raw data can 
be made available upon request to the corresponding author.

\section{Funding}

None

\section{Authors' Contributions}

All authors made substantial contributions to the study. DS, VKS, and PKS conceived and designed the study. DS, PT, BB and HP collected samples, investigated and recorded the laboratory findings at the laboratory. VKS and PKS supervised and provided methods for the study. DS, BB, HP and PC reviewed works of literature and drafted the manuscript. DS, PT, $\mathrm{BB}$ and PCcompiled, curated and interpreted data. DS, PT and DB critically reviewed and revised the manuscript by compiling, formatting, editing and writing the final version of the manuscript. All authors read and approved the final manuscript.

\section{References}

1. Taneja N, Chatterjee S, Singh M, Singh S, Sharma M: Pediatric urinary tract infections in a tertiary care center from north India. Indian J Med Res. 2010 131(1):101-105.

2. Vasudevan R: Urinary tract infection: an overview of the infection and the associated risk factors. J Microbiol Exp 2014, 1(2):42-54.

3. Magliano E, Grazioli V, Deflorio L, Leuci AI, Mattina R, Romano P, et al: Gender and AgeDependent Etiology of CommunityAcquired Urinary Tract Infections. Sci World J. 2012 2012:6.

4. Watson AR, Taylor CM, McGraw M: Forfar and Arneil's Textbook of Pediatrics, 6th edn. Spain: Churchill Livingstone; 2003.

5. National Institute for Health and Care Excellence (NICE): Urinary Tract Infection in Children Diagnosis, Treatment and Longterm Management. NICE Clinical Guidelines, No. 54. In. London: National Institute for Health and Care Excellence (NICE); 2007.

6. Owen D, Vidal-Alaball J, Mansour M, Bordeaux K, Jones KV, Edwards A: Parent's opinions on the diagnosis of children under 2 years of age with urinary tract infection. Fam Pract. 2003 20(5):531-537.

7. Sibi G, Devi AP, Fouzia K, Patil BR: Prevalence, Microbiologic Profile of Urinary Tract Infection and its Treatment with Trimethoprim in Diabetic Patients. Res J Microbio. 2011, 6:543-551.

8. Haghi-Ashteiani M, Sadeghifard N, Abedini M, Taheri-Kalani SSM: Etiology and
Antibacterial Resistance of Bacterial Urinary Tract Infections in Children's Medical Center, Tehran, Iran. Acta Med Iran. 2007 45(2):153-157.

9. Fitzgerald A, Mori R, Lakhanpaul M, Tullus K: Antibiotics for treating lower urinary tract infection in children. Cochrane database Syst Rev. 2012(8).

10. Maduemem KE, Rodriguez YD, Fraser B: How Sensitive are Dipstick Urinalysis and Microscopy in Making Diagnosis of Urinary Tract Infection in Children? Int J Prev Med. 2019 10:62-62.

11. Shaikh N, Mattoo TK, Keren R, Ivanova A, Cui G, Moxey-Mims M, Majd M, Ziessman HA, Hoberman A: Early Antibiotic Treatment for Pediatric Febrile Urinary Tract Infection and Renal Scarring. JAMA Pediatr. 2016 170(9):848854.

12. Cheng Y-W, Wong S-N: Diagnosing symptomatic urinary tract infections in infants by catheter urine culture. J Paediatr Child Health. 2005 41(8):437-440.

13. Subcommittee on Urinary Tract Infection SCoQIaM: Urinary Tract Infection: Clinical Practice Guideline for the Diagnosis and Management of the Initial UTI in Febrile Infants and Children 2 to 24 Months. Pediatrics 2011, 128(3):595-610.

14. Doern CD, Richardson SE: Diagnosis of Urinary Tract Infections in Children. J Clin Microbiol. 2016 54(9):2233-2242.

15. World Health Organization (WHO): Basic laboratory procedures in clinical bacteriology. 2nd edn. Geneva: World Health Organization; 2003.

16. Anígilájé EAB, T. T.: Prevalence and Predictors of Urinary Tract Infections among Children with Cerebral Palsy in Makurdi, Nigeria. Int J Nephrol. 2013 2013:7.

17. Ghorashi Z, Ghorashi S, Soltani-Ahari H, Nezami N: Demographic features and antibiotic resistance among children hospitalized for urinary tract infection in northwest Iran. Infect Drug Resist. 2011 4:171176.

18. Forbes B, Sham D, Weissfeld A: Study Guide for Bailey and Scott's Diagnostic Microbiology. 12th edn: Mosby Elsevier 2007.

19. Ojha AR, Aryal UR: Profile of Children with Urinary Tract Infection and the Utility of Urine Dipstick as a Diagnostic Tool. J Nep Health Res Counc. 2014 12(28):151-155

20. Malla KK, Sarma MS, Malla T, Thapalial A: Clinical Profile, Bacterial Isolates and Antibiotic Susceptibility Patterns in Urinary Tract Infection in Children - Hospital Based Study. J Nepal Paediatr Soc. 2008 28(2):52-61.

21. Brkic S, Mustafic S, Nuhbegovic S, Ljuca F, Gavran L: Clinical and Epidemiology Characteristics of Urinary Tract Infections in Childhood. Med Arh. 2010 64(3):135-138. 
22. Taneja N, Chatterjee SS, Singh M, Sivapriya S, Sharma M, Sharma SK: Validity of Quantitative Unspun Urine Microscopy, Dipstick Test Leucocyte Esterase and Nitrite Tests in Rapidly Diagnosing Urinary Tract Infections. J Assoc Physicians India. 2010 58:485487.

23. Zorc JJ, Kiddoo DA, Shaw KN: Diagnosis and Management of Pediatric Urinary Tract Infections. Clin Microbiol Rev. 2005 18(2):417422.

24. Al-Daghistani HI, Abdel-Dayem M: Diagnostic Value of Various Urine Tests in the Jordanian Population with Urinary Tract Infection. Clin Chem Lab Med. 2005 40(10):10481051.

25. Graham JC, Galloway A: ACP Best Practice No 167: The laboratory diagnosis of urinary tract infection. J Clin Pathol. 2001 54(12):911919.

26. HPA: UK standards for Microbiolog investigations: Investigation of urine. National Standard Method BSOP 41. Standard Units, Microbiology services, Public health England Issue 7. 2009.

27. Lin DS, Huang SH, Lin CC, Tung YC, Huang TT, Chiu NC, et al: Urinary Tract Infection in Febrile Infants Younger Than Eight Weeks of Age. Pediatrics 2000 105(2):e20. 